Bull. Austral. Math. Soc.

$16 \mathrm{~A} 32,16 \mathrm{~A} 38,16 \mathrm{~A} 51$

VOL. 37 (1988) [161-164]

\title{
RINGS IN WHICH EVERY ELEMENT IS THE SUM OF TWO IDEMPOTENTS
}

\author{
Yasuyuki Hirano and Hisao Tominaga
}

\begin{abstract}
Let $R$ be a ring with prime radical $P$. The main theorems of this paper are (1) The following conditions are equivalent: 1) $R$ is a commutative ring in which every element is the sum of two idempotents; 2$) R$ is a ring in which every element is the sum of two commuting idempotents; 3) $R$ satisfies the identity $x^{3}=x$. (2) If $R$ is a PI-ring in which every element is the sum of two idempotents, then $R / P$ satisfies the identity $x^{3}=x$. (3) Let $R$ be a semi-perfect ring in which every element is the sum of two idempotents. If ${ }_{R} R_{R}$ is quasi-projective, then $R$ is a finite direct sum of copies of $G F(2)$ and/or $G F(3)$.
\end{abstract}

Throughout, $R$ will represent a ring with prime radical $P$. A Boolean ring is defined as a ring in which every element is an idempotent. As a generalisation of Boolean rings, we consider the class of rings in which every element is the sum of two idempotents. We begin with an example which shows that such a ring need not be Boolean or even commutative.

Example. Let $A(\neq 0)$ and $B$ be Boolean rings, and $W(\neq 0)$ a $B$ - $A$-bimodule. Assume, furthermore, that $W$ is $s$-unital as a right $A$-module, that is, for any $w$ in $W$, there exists an element $e$ in $A$ such that $w e=w$. Then every element of the non-commutative ring $R=\left(\begin{array}{cc}A & 0 \\ W & B\end{array}\right)$ is the sum of two idempotents. In fact, $\left(\begin{array}{ll}a & 0 \\ w & b\end{array}\right)=\left(\begin{array}{cc}e & 0 \\ w & 0\end{array}\right)+\left(\begin{array}{cc}a-e & 0 \\ 0 & b\end{array}\right)$, where $e$ is an element of $A$ such that $w e=w$.

Our present objective is to prove the following theorems.

THEOREM 1. The following conditions are equivalent:

1) $R$ is a commutative ring in which every element is the sum of two idempotents.

2) $R$ is a ring in which every element is the sum of two commuting idempotents.

3) $\quad R$ satisfies the identity $x^{3}=x$.

Received 20 March 1987

Dedicated to Professor Adil Yaqub on his 60th birthday

Copyright Clearance Centre, Inc. Serial-fee code: 0004-9729/88 \$A2.00+0.00. 
Theorem 2. Let $R$ be a PI-ring in which every element is the sum of two idempotents. Then $R / P$ satisfies the identity $x^{3}=x$.

THEOREM 3. Let $R$ be a semi-perfect ring in which every element is the sum of two idempotents. If $R_{R} R_{R}$ is quasi-projective, then $R$ is a finite direct sum of copies of $G F(2)$ and/or $G F(3)$.

In preparation for proving our theorems, we state four lemmas.

Lемма 1. Let $R(\neq 0)$ be a ring in which every element is the sum of two idempotents. If $R$ contains no non-trivial idempotents, then $R$ is either $G F(2)$ or $G F(3)$.

Proof: Since 0 and 1 are the only idempotents of $R$, we have either $R=\{0,1\}$ or $R=\{0,1,2\}$. Thus $R$ is either $G F(2)$ or $G F(3)$.

LemMA 2. Let $a$ be an element of $R$ with $a^{2}=0$.

(1) If $a=e+f$ for idempotents $e, f$ then $4 a=0$.

(2) If $a=e+f$ for commuting idempotents $e, f$ then $a=2 e$ and $4 e=0$.

Proof: (1) Obviously,

$$
\begin{aligned}
0 & =a^{3}-2 a^{2} \\
& =a+2(e f+f e)+e f e+f e f-2(a+e f+f e) \\
& =e f e+f e f-a .
\end{aligned}
$$

Hence $0=e a^{2} e+f a^{2} f=a+3(e f e+f e f)=4 a$.

(2) Since $0=a^{2}=a+2 e f$, we get $a=-2 e f$, and so $0=a(f-e)=f-e$. Hence $a=2 e$ and $4 e=a^{2}=0$.

LEMMA 3. Let $R$ be a ring with 1 , and $n$ a positive integer greater than 1 . Then the $n \times n$ full matrix ring $M_{n}(R)$ over $R$ contains an element which cannot be written as the sum of two idempotents.

Proof: We write $M_{n}(R)=\sum_{i, j=1}^{n} R e_{i j}$, where $e_{i j}$ are matrix units. Suppose, to the contrary, that every element of $M_{n}(R)$ is the sum of two idempotents. Then, by Lemma $2(1), 4 e_{12}=0$ and so $4 R=0$. Consider the element $a=e_{11}+e_{12}+e_{21}$, and choose idempotents $e=\sum r_{i j} e_{i j}$ and $f$ such that $a=e+f$. Since $a-e=f=$ $f^{2}=a^{2}-a e-e a+e$, we get $a^{2}=a+a e+e a-2 e$. Comparing the coefficients of $e_{11}$, $e_{12}$ and $e_{21}$ on both sides, we get $1=r_{12}+r_{21}, 0=r_{11}+r_{22}-r_{12}$ and $0=r_{11}+$ $r_{22}-r_{21}$, and therefore $1=2 r_{12}$. Then $4 R=0$ implies that $1=4 r_{12}^{2}=0$, which is a contradiction. 
LEMMA 4. Let $R$ be a prime ring in which every element is the sum of two idempotents. If $R \neq Z$, the centre of $R$, then char $R=2$ and $Z$ is either 0 or $G F(2)$.

Proof: First, we claim that $R$ cannot be reduced. Actually, if $R$ is reduced, then every idempotent is central, and so $R=Z$ by hypothesis, a contradiction. Hence $R$ has a non-zero element $a$ with $a^{2}=0$. By Lemma $2(1)$, we conclude that char $R=2$. Now, let $z$ be an arbitrary element of $Z$. By hypothesis, we can write $z=e+f$ for idempotents e, $f$ in $R$. Then it is easily observed that $e f=f e$. Since char $R=2$, we obtain that $z^{2}=e+f+2 e f=e+f=z$. Since $R$ is prime, this implies that $z$ is either 0 or 1 . This completes the proof.

Proof of Theorem 1: 1) $\Longrightarrow 3$ ). It is well-known that $R$ is a subdirect sum of subdirectly irreducible rings $R_{\lambda}$. Since, by Lemma 1 , each $R_{\lambda}$ is either $G F(2)$ or $G F(3), R$ satisfies the identity $x^{3}=x$.

$3)=2$ ). As is well-known, $R$ is a commutative ring. Replacing $x$ by $2 x$ in $x^{3}=x$, we obtain $6 x=0$. Further, replacing $x$ by $x^{2}-x$ in $x^{3}=x$, we obtain $3 x^{2}=3 x$. By making use of these, we see easily that $\left(-2 x^{2}\right)^{2}=4 x^{4}=-2 x^{4}=-2 x^{2}$ and $\left(x+2 x^{2}\right)^{2}=x^{2}+4 x^{3}+4 x^{4}=x^{2}+4 x+4 x^{2}=x+2 x^{2}+3\left(x-x^{2}\right)+6 x^{2}=x+2 x^{2}$. Hence $x$ is the sum of the idempotents $-2 x^{2}$ and $x+2 x^{2}$.

$2) \Longrightarrow 1$ ). Let $a$ be an element of $R$ with $a^{2}=0$. Then, by virtue of Lemma 2 (2), there exists an idempotent $e$ such that $a=2 e$ and $4 e=0$. Now, $-e=f+g$ with some commuting idempotents $f, g$. Then $e=(-e)^{2}=-e+2 f g$, so $2 e=2 f g=2 e f g$. Noting that $f e=e f$, we see easily that $a=2 e=2 e f g=2 e f(-e-f)=-4 e f=0$. Hence $R$ is a reduced ring. As is well-known, every idempotent of the reduced ring $R$ is central, and so $R$ is commutative.

CoROLlaRY 1. Let $R$ be a semiprime ring. If $R$ has the property that every element is the sum of two idempotents, then the centre $Z$ of $R$ has the same property.

ProOF: Since $R$ is semiprime, $R$ is a subdirect sum of prime rings $R_{\lambda}(\lambda \in \Lambda)$. By Lemmas 1 and 4 , the centre $Z_{\lambda}$ of $R_{\lambda}$ is 0 , or $G F(2)$, or $G F(3)$. Now we may regard $Z$ as a subring of the direct product $\prod_{\lambda \in \Lambda} Z_{\lambda}$. Hence $Z$ satisfies the identify $x^{3}=x$. Then, by Theorem 1 , every element of $Z$ is the sum of two idempotents in $Z$.

Proof of Theorem 2: In view of Lemma 1, it suffices to show that every prime factor ring of $R$ is commutative. Suppose, to the contrary, that a prime factor ring $R^{\prime}$ of $R$ is not commutative. By [3, Corollary 1], the ring $Q\left(R^{\prime}\right)$ of central quotients of $R^{\prime}$ is a full matrix ring over a division ring. Then, by Lemma 4, we have that $R^{\prime}=Q\left(R^{\prime}\right)$. Now, Lemmas 1 and 3 force a contradiction that $R^{\prime}$ is either $G F(2)$ or $G F(3)$.

Corollary 2. Let $R$ be an Azumaya $Z$-algebra in which every element is the 
sum of two idempotents. Then $R$ satisfies the identity $x^{3}=x$.

Proof: By [1, Lemma II.3.1], $Z$ is a $Z$-direct summand of $R$, say $R=Z \oplus T$. Then $P=(P \cap Z) \oplus(P \cap Z) T$ by [1, Corollary II.3.7]. As is well-known (see, for example, [1, Theorem II.3.4]), $R$ is a finitely generated $Z$-module, and therefore $R$ is a PI-algebra. Hence, by Theorem $2, R / P$ is commutative. Then, by $[\mathbf{1}$, Proposition II.1.11], we obtain $(P \cap Z) T=T$. Since $P \cap Z$ is a nil ideal of $Z$, and $T$ is a finitely generated $Z$-module, we conclude that $T=0$, and hence $R=Z$. Now, by Theorem $1, R$ satisfies the identity $x^{3}=x$.

Proof of Theorem 3: By [2, Theorem 4.6], $R$ is the finite direct sum of full matrix rings over local rings. Hence, by Lemmas 1 and $3, R$ is the finite direct sum of copies of $G F(2)$ and/or $G F(3)$.

Remark. As is shown in [5] (see also [4]), the following conditions are equivalent:

1) There exists an involution * of $R$ such that $x x^{\star} x=x^{\star}$ for all $x$ in $R$;

2) $R$ is an anti-inverse ring, that is, every element $x$ in $R$ has an anti-inverse $x^{\star} ; x x^{\star} x=x^{\star}$ and $x^{\star} x x^{\star}=x$

3) For each element $x$ of $R$ there exists $x^{\star}$ in $R$ such that $x^{2} x^{\star}=x^{\star}$ and $x^{\star 2} x=x ;$

4) $R$ is a (dense) subdirect sum of fields isomorphic to $G F(2)$ or $G F(3)$

5) $R$ satisfies the identity $x^{3}=x$.

\section{ReFERENCES}

[1] F. DeMeyer and E. Ingraham, 'Separable algebras over commutative rings', in Lecture Note In Math. 181 (Springer-Verlag, Berlin-Heidelberg-New York, 1971).

[2] Y. Miyashita, 'Quasi-projective modules, perfect modules, and a theorem for modular lattices', J. Fac. Sci. Hokkaido Univ. Ser I 19 (1966), 86-110.

[3] L. Rowen, 'Some results on the center of a ring with polynomial identity', Bull. Amer. Math. Soc. $7 \theta(1973), 219-223$.

[4] B.M. Scliein, 'O-rings and LA-rings', Izv. Vyš̌. Učeb. Zaved. Matematika 2(51) (1966), $111-122$. Amer. Math. Soc. Transl. (2) 86 (1970), 137-152. .

[5] H. Tominaga, 'On a theorem of M. Yamada', Proc. 9th Sympos. Semigroups and Related Topics (1985), 40-42. 